\title{
Teilhabe so früh wie möglich
}

\section{Frühförderung und Inklusion}

GITTA PÖTTER

Gitta Pötter ist Leiterin der Überregionalen Arbeitsstelle Frühförderung Brandenburg in Potsdam. www.ffbra.de

\author{
Auch Kinder mit einer Behinderung haben Anspruch \\ auf frühe Förderung und Bildung, wie insbesondere die \\ Behindertenrechtskonvention der Vereinten Nationen als \\ geltendes Recht garantiert. Das System der Frühförderung \\ soll helfen, das Recht auf Teilhabe zu garantieren. \\ Durch eine kontinuierliche Absprachen der Fachkräfte, \\ insbesondere der Frühförder- und Beratungsstellen \\ und der Kindertagesstätten, kann dabei eine hohe \\ Transparenz des jeweiligen Leistungsspektrums und \\ eine abgestimmte Beratung für Eltern erreicht werden.
}

Inklusion bedeutet, dass jeder Mensch, jedes Kind mit und ohne Behinderungen oder drohenden Behinderungen und seine Familie ohne Einschränkungen am Leben in der Gemeinschaft teilhaben kann. Dazu braucht es in der Gesellschaft ein gemeinsames Verständnis zur Bedeutung von Inklusion, über Ziele und Inhalte von Inklusion sowie gemeinsam entwickelte Strategien zur Umsetzung in allen gesellschaftlichen Bereichen. Aus aktueller Sicht ist dies ein langer Weg, der sich insbesondere für Kinder von Geburt bis Schuleintritt lohnt, vertieft zu werden, denn Kinder sind die Quelle und das Spiegelbild unserer Gesellschaft. Inklusion bedeutet »Teilhabe so früh wie möglich«.

»Um den Kindern in ihren Unterschiedlichkeiten Spielraum zur bestmöglichen Entfaltung zu geben, muss der von Ihnen genutzte soziale Raum so gestaltet sein, dass er dieser Vielfalt Rechnung trägt.« (1)

Für Kinder im vorschulischen Alter ist die Familie der wichtigste soziale Raum, in dem sie emotionale Stabilität, Geborgenheit und altersentsprechende Anregungen vorfinden (sollten), um sich mit ihren ureigenen, individuellen inneren Bedingungen weiterzuentwickeln und teilhaben zu können. Durch den Ausbau der Kindertagesbetreuungsangebote für Kinder ab dem ersten Lebensjahr nimmt die Kindertagesstätte für zunehmend jüngere Kinder neben der Familie einen wichtigen sozialen Raum ein. Kinder mit Entwicklungseinschränkungen brauchen auf dem Weg zur Inklusion in diesen beiden wichtigen genannten sozialen Räumen - Familie und Kindertagesstätte - abgestimmte Unterstützungsangebote.

Ein wesentliches Unterstützungsangebot für Familien, die sich um die Entwicklung ihrer Kinder sorgen, bieten auch im Land Brandenburg Frühförderund Beratungsstellen (FFB). Neben einer offenen Anlaufstelle für alle interessierten Eltern bieten die Brandenburger Frühförder- und Beratungsstellen heilpädagogische Diagnostik, eine individuelle und in der Regel heilpädagogische Frühförderung kind- und familienbezogen. Die medizinische Diagnostik wird über die Gesundheitsämter realisiert. Die Brandenburger Frühförder- und Beratungsstellen arbeiten interdisziplinär mit anderen parallel in oder für die $\mathrm{Fa}$ milien tätigen Fachkräften zusammen. Die Finanzierung der Komplexleistung Frühförderung (alle genannten Leistungen aus einer Hand) ist im Land Brandenburg noch nicht umgesetzt.

Durch Kooperationsstrukturen zwischen Frühförder- und Beratungsstellen und Kindertagesstätten realisiert sich die Grundidee einer inklusiven Haltung 
abgestimmt für Eltern und ihre Kinder mit Behinderungen oder drohenden Behinderungen. Dieser Kooperation kommt deshalb eine große Bedeutung zu, weil die Frühförder- und Beratungsstellen Kinder und Familien individuell stärken, damit diese am Leben in der Gemeinschaft (wie z. B. in der Kindertagesstätte) teilhaben können.

\section{Gesetzliche Grundlagen}

Das Übereinkommen der Vereinten $\mathrm{Na}$ tionen über die Rechte von Menschen mit Behinderungen (Behindertenrechtskonvention - BRK) hat das Nachdenken über Teilhabe gesamtgesellschaftlich verstärkt. »Die Behindertenrechtskonvention ist von Deutschland im März 2009 ratifiziert worden und hat damit den Rang eines einfachen Gesetzes erlangt.« (2) Somit ist die Behindertenrechtskonvention seit vier Jahren geltendes Recht in Deutschland und steht gleichrangig neben dem Sozialgesetzbuch I (Allgemeiner Teil), IX (Teilhabe
Gleichwohl das SGB IX als erstmalige Vorgabe ermöglicht, traditionell unterschiedliche, fachliche, pädagogisch-psychologische und medizinischtherapeutische Angebote und Hilfen in eine Zuständigkeit zusammenzuführen, besteht darüber hinaus die Notwendigkeit, dass die verschiedenen Rehabilitationsträger sich auf ein gemeinsames fachliches, administratives und Refinanzierungs-Konzept verständigen müssen. Nur dann kann die Teilhabe von Kindern und die Bereitstellung von notwendigen Leistungen der medizinischen Rehabilitation gleichermaßen sichergestellt werden.

Das System der Frühförderung erhielt durch das SGB IX und tiefgründiger durch die Frühförderungsverordnung erstmalig deutliche gesetzliche Grundlagen $(\mathbb{S} 3$ FrühV) und unterstützt die Realisierung von Teilhabe über einen interdisziplinären Förder- und Behandlungsplan $(\mathbb{S} 7$ FrühV) für jedes Kind mit Behinderungen oder drohenden Behinderungen und seine Familie. Eine $\mathrm{Zu}$ -

\section{"Kinder mit Entwicklungseinschränkungen brauchen sowohl in der Familie wie}

\section{in der Kindertagesstätte abgestimmte Unterstützungsangebote"}

und Rehabilitation) und den Sozialgesetzbüchern XII (Sozialhilfe) und VIII (Jugendhilfe), in denen die Eingliederungshilfe für Menschen mit Behinderungen geregelt wird.

Die Angebote der Frühförderung und der Kindertagesbetreuung können den Artikeln 24 und 26 in der Behindertenrechtskonvention und der frühkindlichen Bildung zugeordnet werden. Hier steht das Recht auf inklusive und hochwertige Bildung als Grundlage für Chancengleichheit. Niemand darf aufgrund von Behinderungen (oder sonstigen »Auffälligkeiten «) vom allgemeinen Bildungssystem ausgeschlossen werden. Zur Verwirklichung dieser Ziele verpflichten sich die Vertragsstaaten u. a., "angemessene Vorkehrungen für die Bedürfnisse des Einzelnen« zu treffen, »lebenspraktische Fertigkeiten« zu vermitteln (am einzelnen Kind und seiner Familie ausgerichtet) und alle dazu "notwendigen und geeigneten Maßnahmen« zu ergreifen. sammenführung medizinisch-therapeutischer und pädagogisch-psychologischer Leistungen ( $\mathbb{S} \mathbb{S} 5,6$ FrühV) wird bei Bedarf möglich. Durch kontinuierliche Absprachen der Fachkräfte, insbesondere der Frühförder- und Beratungsstellen und Kindertagesstätten, kann eine hohe Transparenz des jeweiligen Leistungsspektrums und eine abgestimmte Beratung für Eltern erreicht werden.

»Die Teilhabe ist dabei gegeben, wenn eine Person sozial eingebunden ist, d. h., wenn individuelle und umweltbezogene Faktoren es ermöglichen, dass die Person die sozialen Rollen, die ihr wichtig und ihrer Lebenssituation angemessen sind (z. B. in der Familie, in der Kindertagesstätte), auch einnehmen und zu ihrer Zufriedenheit ausfüllen kann.« »Damit ist für die Weiterentwicklung der Rehabilitation eine interdisziplinäre Berücksichtigung der Kontextfaktoren von entscheidender Bedeutung. Die gezielte Förderung der Teilhabe und Selbstbe- stimmung als zentrale Zielsetzung der Rehabilitation kann somit nur gelingen, wenn die vielfältigen Zusammenhänge zwischen Gesundheitsproblem und Kontextfaktoren angemessen verstanden und bei der Planung von Interventionen einbezogen werden.« (3)

Dabei unterstützt die Anwendung der Internationalen Klassifikation der Funktionsfähigkeit, Behinderung und Gesundheit für Kinder (ICF-CY) das Finden einer gemeinsamen Sprache für die Beschreibung funktionaler Gesundheit zwischen den Fachleuten im Gesundheits- und Sozialwesen (BAG für Reha 2008).

\section{Frühförderung und inklusive Entwicklung}

Die Umsetzung von Teilhabe kann im frühkindlichen Bereich aus dem BalanceModell von Otto Speck abgeleitet werden (vgl. Abb. Seite 144). Dieses Balance-Modell illustriert, was sozialethisch, pädagogisch, psychologisch und organisatorisch in der Auseinandersetzung mit Inklusion und Teilhabe in der frühkindlichen Bildung beachtet und in ständig neue Balance gebracht werden muss.

Soziale Teilhabe im Kindergruppenalltag realisiert sich nur über individuell gestärkte Ressourcen jedes einzelnen Kindes. Die Frühförder- und Beratungsstellen sind Institutionen, die durch ihren Anspruch der individuellen Begleitung der Eltern und des jeweiligen Kindes mit Behinderungen oder drohenden Behinderungen das familiäre System stärken und dem Kind Entwicklungsimpulse geben. Damit kann dieses sich letztendlich auch mit seinen entsprechenden Bedingungen weiterentwickeln. Das Kind stärkt seine inneren Voraussetzungen, um in der Kita und im familiären Umfeld an den Aktivitäten des täglichen Lebens teilhaben zu können. Das bedeutet, dass durch die Arbeit der Frühförder- und Beratungsstellen besonders im Beratungs-, Diagnostik- und im Förderprozess selbst dem Rehabilitationsbegriff Artikel 24 und 26 der UN-Behindertenrechtskonvention entsprochen wird, weil ein höchstes Maß an Unabhängigkeit durch die Frühförderung erreicht wird. Somit stärken die Angebote der Frühförder- und Beratungsstellen die individuelle Selbstentfaltung und Selbstbestimmung der Kinder und der Familien. Eine soziale Teilhabe in der Kindertagesstätte wird möglich. $\rightarrow$ 


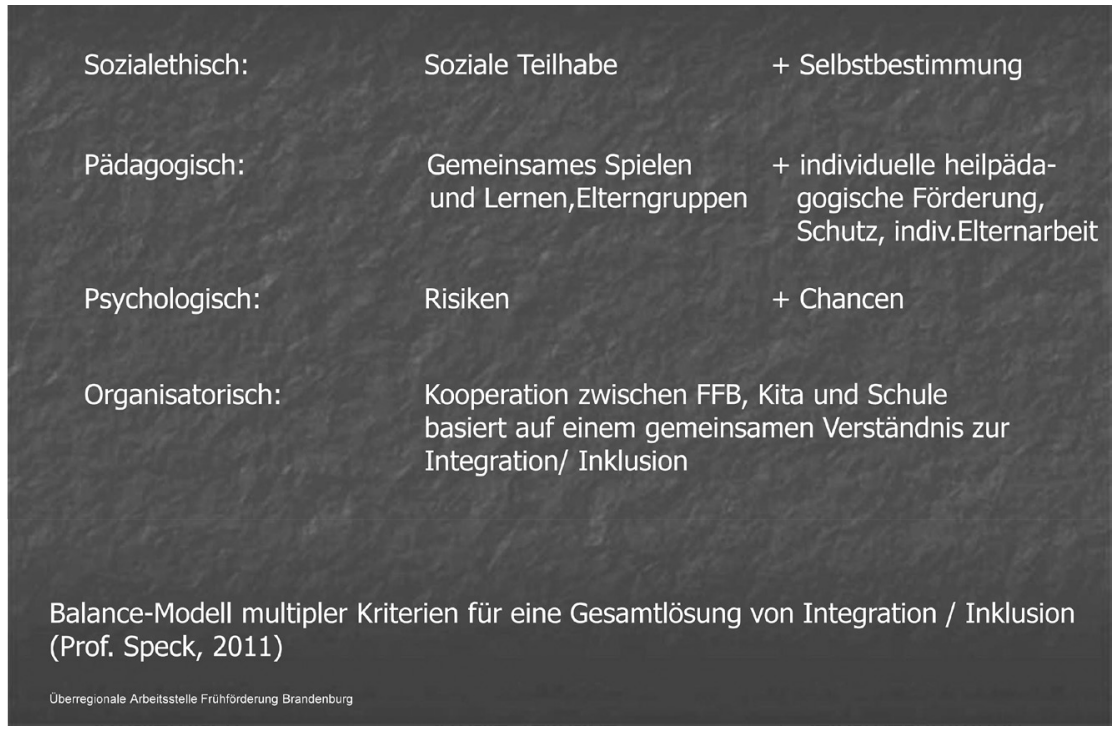

Mit einem Modell kann gezeigt werden, was in der Auseinandersetzung mit Inklusion und Teilhabe in der frühkindlichen Bildung immer beachtet und in ständig neue Balance gebracht werden muss.

\section{Herausforderungen an die Frühförderung}

Es braucht eine Klarstellung, dass Frühförder- und Beratungsstellen teilhabeorientiert arbeiten und die Inklusionsprozesse hauptsächlich in Familien und im Wirkungsort Kindertagesbetreuung unterstützen können, genauso wie im Prozess der Überleitung zur Schule. Innerhalb der Frühförder- und Beratungsstellen muss das »Kerngeschäft « zur Umsetzung dieses inklusiven Ansatzes sichergestellt sein: Die fachlichen Anforderungen von Frühförderung müssen weiterhin leistbar bleiben: Ganzheitlichkeit, Familienorientierung, Mobilität, Interdisziplinarität und Lebensweltorientierung.

Nur wenn die unterschiedlichen fachlichen Akteure einheitlich in und mit Familien wirken, fühlt die Familie mit ihrem Kind sich bestärkt und kann letztendlich gestärkt am täglichen Leben teilnehmen, also Teilhabeleistungen wahrnehmen. Um die Schnittstellen zwischen »Auf dem Weg zur Inklusion « und der »Weiterentwicklung von Frühförderung « beschreiben zu können, braucht es eine kontinuierliche Qualifizierung der Leitungskräfte und Mitarbeiterinnen der Frühförder- und Beratungsstellen.

Zur Qualitätssicherung und Weiterentwicklung sind Qualitätsstandards für die Offene Beratung und Erstberatung, Interdisziplinäre Diagnostik, Besonderheiten der Frühförderarbeit (pädagogisch, therapeutisch) in Fami- lien - insbesondere für Kinder mit psychosozialen Risiken - zu beschreiben.

Die Vernetzungsaktivitäten zwischen den Akteuren (Leistungsträger und Leistungserbringer) müssen entsprechend der Bedürfnisse von den Familien zur Teilhabe thematisiert und lösungsorientiert auf den Weg gebracht werden (Sozialraumorientierung). Bewährt haben sich regionale Arbeitskreise zur Frühförderung, in denen Leistungsträger und Leistungserbringer einen kontinuierlichen Erfahrungsaustausch pflegen und Fachpapiere als gemeinsame Arbeitsgrundlage entwickeln. (4)

Beachtung finden muss in diesem Kontext die Weiterentwicklung der Eingliederungshilfe auch entsprechend des 13. Kinder- und Jugendberichtes für die Ausgestaltung der Komplexleistung Frühförderung. Hierbei ist wichtig, dass die Einbettung der Eingliederungshilfe in die Jugendhilfe nur dann eine sinnvolle Lösung wäre, wenn zur Umsetzung und Sicherstellung der Komplexleistung Frühförderung die Krankenkassenleistungen (SGB V) mitgedacht werden und diese Akteure von Anfang an für den Bereich der Komplexleistung Frühförderung in das Gedankengut der »Großen Lösung « eingebettet werden.

Das gemeinsame Wirken der Frühförderung mit den Angeboten der »Frühen Hilfen « ist im Zeitalter der Inklusion von Bedeutung. Die Zusammenarbeit zwischen den Brandenburger »Netzwerken Gesunde Kinder« und den Frühförder- und Beratungsstellen zur frühzeitigen Früherkennung von Entwicklungsstörungen sollte kontinuierlich und finanziert fortgesetzt werden. Dazu ist die Definition der Inhalte von Frühen Hilfen (u. a. projektbezogen, als Bestandteil des Kinderschutzgesetzes, z. B. Familienhebammen) im Verhältnis zu den Inhalten der Frühförderung notwendig.

Fazit: Die Umsetzung der Inklusion im frühkindlichen Bereich braucht Rahmenbedingungen.

\section{Anmerkungen}

(1) LAG Frühe Hilfen in Hessen: Was Kinder im Rahmen einer inklusiven Tagesbetreuung benötigen. Eine Handreichung zu Anforderungen und Gestaltungsmöglichkeiten für Fachkräfte von Kindertageseinrichtungen und Frühförderstellen.

(2) Klaus Lachwitz, aus dem Vortrag »Fachgespräch Frühförderung und Inklusion« in Potsdam, Dezember 2011.

(3) Farin-Glattacker, Erik, AG Teilhabeforschung 2011.

(4) Regionale Arbeitskreise Frühförderung - Kooperation und Vernetzung im Brandenburger Frühfördersystem. Erfahrungsbericht, Überregionale Arbeitsstelle Frühförderung Brandenburg 2010.

\section{Literatur}

Farin-Glattacker, Erik: AG Teilhabeforschung, 2011 Hinz, Andreas; Körner, Ingrid; Niehoff, Ulrich: Von der Integration zur Inklusion, hg. von der Bundesvereinigung Lebenshilfe für Menschen mit geistiger Behinderung e.V., 2008.

Lachwitz, Klaus, aus dem Vortrag "Fachgespräch Frühförderung und Inklusion« in Potsdam, Dezember 2011.

Landesarbeitsgemeinschaft Frühe Hilfen e. V.: Was Kinder im Rahmen einer inklusiven Tagesbetreuung benötigen. Eine Handreichung zu Anforderungen und Gestaltungsmöglichkeiten für Fachkräfte von Kindertageseinrichtungen und Frühförderstellen, 2012.

Speck, Otto: Balance-Modell multipler Kriterien für eine Gesamtlösung von Integration/ Inklusion, 2011.

Überregionale Arbeitsstelle Frühförderung Brandenburg: Regionale Arbeitskreise Frühförderung. Kooperation und Vernetzung im Brandenburger Frühfördersystem. Erfahrungsbericht, 2010. 